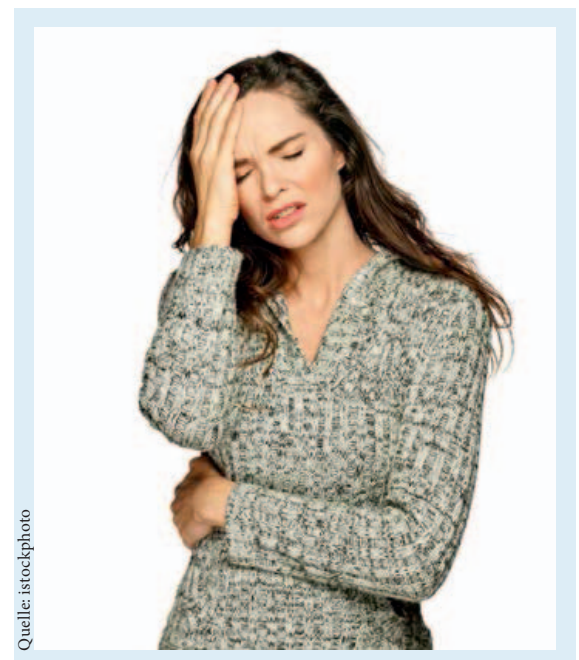

\section{Chronische Erkrankungen und Multimorbidität}

Das Leben fordert Tribut von der Gesundheit. Viele chronische Erkrankungen sind Zivilisationskrankheiten. Mit der «Lifestyle Medicine» macht sich die Schulmedizin einen bewährten Ansatz der Komplementärmedizin zu eigen, den Lebenswandel des Patienten in die Prävention und Behandlung einzubeziehen. In den Expertengesprächen erfahren Sie, wie sich chronische Erkrankungen behandeln lassen und wie man hilft, sie zu vermeiden: Ein naturgemässes Leben spielt dabei eine wichtige Rolle.

\title{
Die richtige Lebensführung für mehr Gesundheit
}

«Lifestyle Medicine» ist seit etwa anderthalb Jahrzehnten ein neuer Trend in der Medizin. Ähnlich wie die Ganzheitsmedizin begreift er die Lebensumstände des Menschen als entscheidend für die Gesundheit.

Chronische Erkrankungen sind oft solche, die sich auf jene Lebensgewohnheiten zurückführen lassen, die gesundheitsgefährdend sind. Das moderne Leben, die Arbeitsbedingungen, das Freizeitverhalten, die Ernährung und die Konsumgewohnheiten bringen sie mit sich. Nicht von ungefähr zählen z.B. Erkrankungen des Muskelsystems, wie sie z.B. bei Rückenschmerzen zu beobachten sind und durch schlechte Haltung am Arbeitsplatz verursacht werden, zu den häufigen chronischen Erkrankungen, mit denen sich Mediziner beschäftigen müssen. Auch psychische Beschwerden, etwa ein BurnoutSyndrom, oder ADHS (Aufmerksamkeitsdefizit-/Hyperaktivitätsstörung) bei Kindern, Jugendlichen und auch Erwachsenen sind langwierig und können oft auf die Lebensführung zurückgeführt werden.

Das moderne Leben hat seinen Preis

Etwa 70\% der Erkrankungen gehen auf den Lebensstil zurück. In den USA entfallen 80\% der Gesundheitskosten auf die Behandlung von Faktoren von Krankheiten, die durch einen falschen Lebensstil hervorgerufen werden.

Das gesamte Leben des Patienten steht bei der Komplementärmedizin seit jeher im Mittelpunkt des ärztlichen Interesses. In den letzten 1015 Jahren hat sich in der Medizin ein neuer Ansatz etabliert, mit dem ähnlich umfassend wie in der Komplementärmedizin chronische Zivilisationskrankheiten zu Leibe gerückt werden soll. Mit der «Lifestyle Medicine» setzt sich auch in der Schulmedizin die schon seit Langem von der Komplementärmedizin vertretene Erkenntnis durch, dass die Umwelt eine wesentliche Rolle bei der Erhaltung der Gesundheit spielt. Sie versteht sich ausdrücklich als umfassendes Behandlungs- und Präventionskonzept für chronische Erkrankungen, die auf die Zivilisation zurückzuführen sind. Sie gehören $\mathrm{zu}$ den häufigsten Todesursachen weltweit; zu den Ursachen zählen z.B. chronischer Stress, das Rauchen, eine falsche Ernährung, Inaktivität, Alkoholmissbrauch oder auch soziale Faktoren wie z.B. Vereinsamung. $\mathrm{Zu}$ den klassischen chronischen Lifestyle-Erkrankungen zählen z.B. Herzkreislauferkrankungen, Übergewicht, Bluthochdruck, Diabetes mellitus Typ 2, bestimmte Formen von Krebs oder Stoffwechselstörungen sowie Muskelerkrankungen.

Mediziner, die sich auf die Ganzheitsmedizin spezialisiert haben, finden im Ansatz der «Lifestyle Medicine» interessante Denkanstösse und Konzepte. Ebenso finden Vertreter der Schulmedizin innerhalb der «Lifestyle Medicine» im komplementärmedizinischen Ansatz befruchtende Elemente. Die «Lifestyle Medicine» sorgt dafür, dass sich die Schulmedizin vom Schwerpunkt der ausschliesslich medikamentösen Behandlung, 


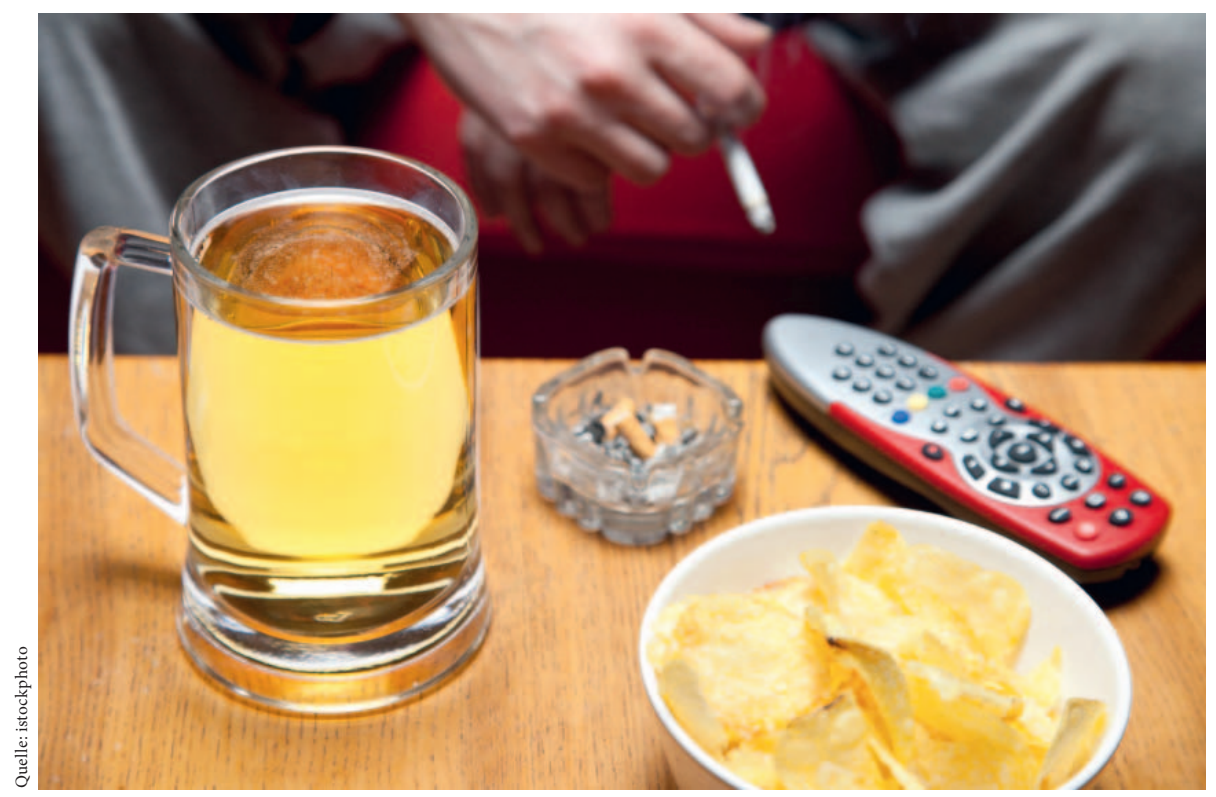

die sich auf die direkte Kausalität konzentriert, löst und den Patienten in seinem Leben umfassend wahrnimmt und ihn auch entsprechend behandelt. Eine Erkrankung hat nach diesem Verständnis vielfältige Ursachen, die auch in einer pathologischen Lebensführung begründet sein können. Diese Erkenntnis, die sich jetzt in der Schulmedizin durchzusetzen beginnt, könnte auch auf die Anerkennung der Komplementärmedizin abfärben, denn die «Lifestyle Medicine» versteht sich als durch und durch klassische schulmedizinische Disziplin.

\section{Ein neues Feld in der Medizin}

Die Geburtsstunde der «Lifestyle Medicine» war die Publikation des gleichnamigen Lehrbuchs von James Rippe im Jahr 1999 [1], das seitdem zu vielen interessanten Ansätzen bei der Behandlung von Zivilisationskrankheiten geführt hat. Im Zentrum der Behandlung des Patienten steht dabei die Veränderung von dessen Lebensweise. Um dies zu erreichen, beschränken sich die Ärzte aber nicht auf Ermahnungen wie «Trinken Sie nicht so viel Alkohol» oder «Bewegen Sie sich wieder mehr» oder «Stellen Sie Ihre Ernährung um».
In ihnen entsteht durch den sozialen Druck eine grössere Motivation, das eigene Verhalten entsprechend $\mathrm{zu}$ ändern und diese Änderung auch langfristig beizubehalten. Die medikamentöse Behandlung wird während der Therapie allerdings aufrechterhalten. In vielen Fällen muss sie jedoch modifiziert werden, da die Dosierung durch die Veränderung des Lebensstils möglicherweise zu hoch oder ganz falsch ist. Die gesündere Lebensführung wirkt sich sehr schnell auf den Bedarf an weiteren Medikamenten aus, der stets zurückgeht. So kann es notwendig sein, die Insulingabe bei Patienten mit Diabetes mellitus Typ 2 anzupassen, weil sie ihre Ernährungsgewohnheiten verändern. Zum Teil kann Insulin dann ganz abgesetzt oder durch Metformin/Thiazolidindione oder Sulfonylurea ersetzt werden. Bei längeren Behandlungsdauern sind die Erfolge einer Veränderung der Lebensführung wesentlich besser, wenn sie durch Medikamente unterstützt werden. So ist etwa die Raucherentwöhnung zwischen zwei- und dreimal so erfolgreich, wenn sie durch ein Bupropion unterstützt wird. Die wissenschaftliche Evidenz zeigt, dass die "Lifestyle Medicine» sich durchaus eignet, um chronische Zivilisationskrankheiten zu behandeln bzw. sie erst gar nicht auftreten zu lassen. So gibt es etwa eine Evaluation der «Lifestyle Medicine» bei der Prävention von Diabetes. In einer multizentrischen Studie [2] wurde untersucht, ob ein leichter Gewichtsverlust durch eine veränderte Ernährung oder durch vermehrte körperliche Aktivitäten oder nur durch die Behandlung mit Metformin den Ausbruch von Diabetes verhindern oder zumindest herauszögern kann. Die Patienten, die an der Studie teilnahmen, hatten alle über dem Durchschnitt liegende Glukosewerte, die allerdings noch nicht so hoch waren, als dass man eine Diabetes-Diagnose hätte stellen können. Es zeigte sich, dass das Risiko, an Diabetes mellitus 
Typ 2 zu erkranken, bei den Patienten, die es schafften, ihr Gewicht zu verringern, stark sank. Der Effekt war grösser als bei einer medikamentösen Behandlung mit Metformin [2]. Die Veränderungen, die bereits einen grossen Erfolg mit sich brachten, waren vergleichsweise gering. Weniger Fett und weniger Kalorien zu sich zu nehmen und sich etwa zweieinhalb Stunden pro Woche körperlich $\mathrm{zu}$ bewegen, sorgten bereits dafür, dass das Gewicht um etwa 7\% sank und auch auf diesem Stand blieb. Die Verhaltensänderung war nur deshalb so erfolgreich, weil die Patienten ein intensives Verhaltenstraining, wie sie ihre Gewohnheiten nachhaltig verändern können, absolvieren mussten.

Erfolgreich ist der Ansatz auch bei der Bekämpfung des Übergewichts. Eine Kombination von mehr Bewegung und eine Umstellung der Ernährung brachte eine grössere Gewichtsabnahme als lediglich eine leichte Umstellung der Diät [3]. Die erste Gruppe hatte fast $11 \mathrm{~kg}$, die zweite Gruppe nur etwa 8 kg verloren. Auch hier waren die Umstellung von Ernährung und die vermehrte Bewegung von einem Therapieprogramm begleitet. Auf lange Sicht können Programme, die die Lebensweise verändern, ebenfalls Erfolg haben. Ein Beispiel hierfür sind etwa Therapien, mit denen Kinder zu einer gesünderen Lebensführung bewegt werden. Sie zielen etwa darauf $a b$, den Cholesterinspiegel zu senken und damit das Risiko der Kinder zu minimieren, im Erwachsenenalter einmal an einer Herzkreislauferkrankung zu leiden. In einer retrospektiven Untersuchung an 539 Kindern, die 1985 entweder 9, 12 oder 15 Jahre alt waren, zeigte sich bei einer erneuten Untersuchung 20 Jahre später, dass sich Anstrengungen, den Lebenswandel zu ändern, in einer verbesserten Gesundheit niederschlugen. Viele Kinder, die vor 2 Jahrzehnten gute Aussichten auf eine typische Krankheitskarriere als Übergewichtige hatten, waren als Erwachsene gesund. Sie hatten es geschafft, durch einen gesunden Lebenswandel das Übergewicht als Risikofaktor loszuwerden und sich auch sonst einen besseren und gesünderen Lebensstil zuzulegen. Ein erhöhtes Risiko für Herzkreislauferkrankungen hatten jene Kinder, die in der Zwischenzeit begonnen hatten $\mathrm{zu}$ rauchen oder körperlich ziemlich inaktiv geworden waren. Eine wichtige Rolle spielt scheinbar auch der sozioökonomische Status, den die Kinder im Laufe ihres Lebens erreicht haben und der natürlich auf den Lebensstil einen grossen Einfluss hat. Je niedriger dieser war, desto grösser war das Gesundheitsrisiko.

\section{Weniger Stress, weniger psychische Erkrankungen}

Verhaltensänderungen sorgen aber nicht nur bei somatischen Erkrankungen für ein geringeres Risiko, sondern auch bei psychischen Erkrankungen und Beeinträchtigungen. Besonders Stress scheint sich durch die bewusste Veränderung der Lebensweise reduzieren $\mathrm{zu}$ lassen. Es gibt Studien, die zeigen, dass z.B. regelmässige Meditation zu einer Verringerung von Stress führt. Coaching, das zu einer besseren Planung der Arbeit und einer stärkeren Trennung von Beruf und Freizeit führt, scheint ebenfalls geeignet $\mathrm{zu}$ sein, um die Anspannung abzubauen, unter der viele Menschen heute leiden. Auch schwere psychische Erkrankungen wie z.B. Angststörungen scheinen sich bekämpfen, therapieren und teilweise verhindern zu lassen, wenn die Patienten es schaffen, Risikofaktoren in ihrem Leben zu beseitigen oder sie zu minimieren. So gibt es Hinweise, dass im Falle von Angststörungen ein Training, das hilft, das Vertrauen in die eigene physische Widerstandskraft zu steigern, dafür sorgt, dass das Risiko, an einer Angststörung zu leiden, abnimmt.

Ein gutes Hilfsmittel, um Verhaltensänderungen bei den Patienten langfristig $\mathrm{zu}$ etablieren, scheinen Apps für Smartphones zu sein [4]. Sie helfen, den Lebensstil nachhaltig $\mathrm{zu}$ verändern. In den USA sind z.B. Abnehmprogramme sehr beliebt, bei denen der soziale Druck, sie auch wirklich durchzuziehen, eine wichtige Rolle spielt. Mit einem App sind die Patienten mit ihrer sozialen Gruppe im Netz verbunden und stehen so unter einem gewissen Druck, sich nicht von ihrem Ziel abbringen $\mathrm{zu}$ lassen, sondern auf ihrem Weg weiter fortzuschreiten und ihren Lebenswandel so umzustellen, dass er ihrer Gesundheit dient. Eine reelle Therapiegruppe wird dabei durch «Social Media» stärker. Mit diesem Ansatz, die Lebensführung als entscheidend für die Prävention und Behandlung vieler chronischer Erkrankungen $\mathrm{zu}$ erkennen, trifft die Schulmedizin von einer ganz anderen Seite kommend auf die Erkenntnis, die es in der Ganzheitsmedizin schon seit Langem gegeben hat, dass die Lebensführung sehr entscheidend für die Gesundheit des Menschen ist und dass, wenn man die Gesundheit der Patienten verbessern will, man sich intensiv um deren Lebensführung kümmern muss. (rfi)

\section{Literatur}

1 Rippe J: Lifestyle Medicine, ed 2. New York, Taylor \& Francis, 2013.

2 Knowler WC, et al.: Reduction in the incidence of type 2 diabetes with lifestyle intervention or metformin. N Engl J Med 2002;346:393-403.

3 Goodpaster $\mathrm{BH}$, et al.: Effects of diet and physical activity interventions on weight loss and cardiometabolic risk factors in severely obese adults: a randomized trial. JAMA 2010;304:1795-1802.

4 Freedman DH: The Perfect Self. The Atlantic 2012;42-52. 